\title{
The Emerging Roles of Extracellular Vesicles As Communication Vehicles within the Tumor Microenvironment and Beyond
}

\author{
Ryan Sullivan', Grace Maresh', Xin Zhang ${ }^{1}$, Carlos Salomon ${ }^{2,3,4}$, John Hooper, \\ David Margolin ${ }^{6,7}$ and $L i L^{1,7 *}$
}

'Laboratory of Translational Cancer Research, Ochsner Clinic Foundation, New Orleans, LA, United States, ${ }^{2}$ Exosome Biology Laboratory, Centre for Clinical Diagnostics, University of Queensland Centre for Clinical Research, Royal Brisbane and Women's Hospital, The University of Queensland, Brisbane, QLD, Australia, ${ }^{3}$ Maternal-Fetal Medicine, Department of Obstetrics and Gynecology, Ochsner Clinic Foundation, New Orleans, LA, United States, ${ }^{4}$ Faculty of Pharmacy, Department of Clinical Biochemistry and Immunology, University of Concepción, Concepción, Chile, ${ }^{5}$ Mater Research Institute-University of Queensland, Brisbane, QLD, Australia, ${ }^{6}$ Department of Colon and Rectal Surgery, Ochsner Clinic Foundation, New Orleans, LA, United States, ${ }^{7}$ Ochsner Clinical School, School of Medicine, University Queensland, New Orleans, LA, United States

\section{OPEN ACCESS}

Edited by:

Giovanna Mantovani, Università degli Studi di Milano, Italy

Reviewed by: Erika Peverelli,

Università degli Studi di Milano, Italy

Manuel D. Gahete, Instituto Maimónides de Investigación Biomédica de Córdoba, Spain

*Correspondence:

Ili@ochsner.org

Specialty section: This article was submitted to Systems and Translational Endocrinology, a section of the journal Frontiers in Endocrinology

Received: 31 May 2017

Accepted: 25 July 2017 Published: 08 August 2017

Citation:

Sullivan R, Maresh G, Zhang X, Salomon C, Hooper J, Margolin D and Li L (2017) The Emerging Roles of Extracellular Vesicles As Communication Vehicles within the Tumor Microenvironment and Beyond.

Front. Endocrinol. 8:194. doi: 10.3389/fendo.2017.00194
Tumors evolve in complex and dynamic microenvironments that they rely on for sustained growth, invasion, and metastasis. Within this space, tumor cells and non-malignant cells are in frequent communication. One specific mode of communication that has gained recent attention is the release of extracellular vesicles (EVs). EVs are lipid bilayer-bound vehicles that are released from the cell membrane and carry nucleic acids, proteins, and lipids to neighboring or distant cells. EVs have been demonstrated to influence a multitude of processes that aid in tumor progression including cellular proliferation, angiogenesis, migration, invasion, metastasis, immunoediting, and drug resistance. The ubiquitous involvement of EVs on cancer progression makes them very suitable targets for novel therapeutics. Furthermore, they are being studied as specific markers for cancer diagnostics, prognosis, and even as chemotherapy drug-delivery systems. This review focuses on the most recent advances in EV knowledge, some current and potential problems with their use, and some proposed solutions to consider for the future.

Keywords: extracellular vesicle, exosome, microvesicle, cancer, tumor microenvironment

\section{INTRODUCTION}

Cancer is a leading cause of morbidity and mortality worldwide. In 2015, it was responsible for 8.8 million deaths, making it the second leading cause of death globally (1). In the United States, nearly $40 \%$ of people will be diagnosed with cancer within their lifetimes, and the national expenditure for cancer patient care is estimated to reach $\$ 156$ billion by the year 2020 .

A great deal of progress has been made in understanding cancer development over the last century enabling a steady decline in cancer mortality over the past two decades (2). However, in general, there still remains a large gap in survival rates between localized and metastatic disease (3), indicating that advanced disease is more poorly understood and more difficult to treat. In fact, early detection still remains one of the most reliable ways to ascertain an effective treatment response (4). The high mortality of advanced disease calls for a better understanding of cancer cell biology and, in particular, the mediating factors leading to cancer progression. One such realm of cancer biology that is increasingly recognized as a key factor in tumor progression, metastasis, and chemotherapeutic drug resistance is the tumor microenvironment (TME) (5). 
Tumors develop in complex and dynamic environments that aid their sustained growth, invasion, and metastasis (6). This space, called the TME, directly surrounds the tumor and consists of an array of non-malignant cells including fibroblasts, immune cells, adipose tissue, blood and lymphatic vessel networks, and signaling molecules of the extracellular matrix. Current research has demonstrated the vital role of the TME in maintaining and progressing the tumor phenotype and as a possible focus for targeted therapy (6). Complex interactions between the TME and malignant cells occur through a very sophisticated network of cellular communication. Many of these signaling pathways operate through direct cell-to-cell contact or by classical paracrine signaling loops of cytokines or growth factors with their receptors. However, more recently, extracellular vesicle (EV) shedding has emerged as another important mechanism of cellular interchange (7). EVs are lipid bilayer-bound vehicles that are released from the cell membrane and carry nucleic acids (DNA, mRNA, and miRNA), proteins, and lipids to neighboring or distant cells (8). EVs exhibit wide-ranging roles in maintaining normal cellular and biological physiology. Undoubtedly, however, the most heavily researched area of EV-associated pathology is their role in tumor development and chemotherapeutic resistance in cancer. In this review, we highlight the most current studies involving EVs and their influence on the pathogenesis of cancer.

\section{THE TME}

Cells reside in diverse microenvironments that help maintain physiological order within a tissue. During tumorigenesis, this region consists of tumor cells and an array of non-malignant cells including fibroblasts, immune cells, adipose tissue, blood and lymphatic vessel networks, and the extracellular matrix called the TME (5). In fact, non-cancerous cells can account for greater than $50 \%$ of the overall tumor mass (9). Within the TME, complex communication pathways are occurring among its inhabitants (10), allowing the tumor and the TME to independently influence one another and co-evolve. It is apparent that the TME has a vital role in maintaining and progressing the tumor phenotype and that it is a possible focus for targeted therapy (6).

A healthy local microenvironment surrounding a tumor may initially help to provide anticancer effects. This was first demonstrated in melanoma where the number of tumor-infiltrating lymphocytes was shown to correlate with better clinical outcomes (11). This concept has also been demonstrated in many other malignancies including colorectal cancer, where an active TME and signs of an immune response were associated with the absence of early metastasis and increased survival (12). In fact, the notion that local immune cells act as guards against nascent transformed cells was developed over 60 years ago (13).

However, as tumors develop, their microenvironment becomes disrupted, protection is lost, and tumor progression may be allowed to continue. The association between a disrupted microenvironment and malignancy is well established and is illustrated during the process of chronic inflammation (14) where local dysfunction acts to induce oncogenic mutations, genomic instability, and early tumor promotion and enhance angiogenesis (14). Tumors can directly disrupt and manipulate their local microenvironment by hijacking local cells and coercing them to provide varying services to help avoid the immune response or aid in other processes of tumor development by supplying cytokines, growth factors, and proteinases (15). Perhaps the best studied of these commandeered cells are the tumor-associated macrophage (TAM), the cancer-associated fibroblast (CAF), and the myeloid-derived suppressor cells (MDSCs).

The TAM is a pirated cell of the TME that provides local support to the tumor (16). In normal circumstances, macrophages act as a main defense against pathogens and a bridge between the innate and adaptive immune systems. As they become immersed in the evolving TME and are exposed to hypoxic conditions and tumor-derived factors, macrophages begin to change phenotype and functionality (17). This change may have serious implications for patient prognosis. A large array of clinical data indicate that the accumulation of TAMs within a tumor is associated with poorer outcomes (18). The production of TAMs was originally described as a phenotypic change of the M1 macrophage, which produces pro-inflammatory cytokines, to the M2 macrophage, known to produce anti-inflammatory and protumorigenic functions (19). This has been recently challenged in a mouse mammary cancer model that suggested TAMs are phenotypically and functionally different from M2 macrophages. The study further reports that TAMs more likely originate from CCR2+ inflammatory monocytes that depend on the notch signaling pathway for differentiation (20).

Cancer-associated fibroblasts are fibroblasts that have become activated by local factors within the TME (21). They have similar morphological properties to myofibroblasts (22) and assume the phenotype of a facilitator of tissue repair by generating and releasing growth factors and regulating inflammation and immunity (23). CAFs, unlike normal physiologically activated fibroblasts, are constitutively active and neither revert to a normal phenotype nor undergo apoptosis (24). They have been implicated in many aspects of tumor progression through varying mechanisms (25). For example, when CAFs isolated from human breast carcinoma were subcutaneously co-injected with a breast cancer cell line into an immunodeficient murine host, tumor proliferation was significantly increased in the presence of CAFs compared to normal mammary fibroblasts isolated from the same patient. Further experiments attributed this to the CAF's ability to secrete stromal cell-derived factor-1 (SDF-1; also known as CXCL12) (26). It has also been shown that mice orthotopically co-xenografted with human pancreatic cancer cells and CAFs develop pancreatic tumors and metastases and that the CAF secretome stimulates the epithelial-mesenchymal transition (EMT) (27). CAFs also assist in metastasis by supplying transforming growth factor beta (TGF- $\beta$ ) to tumor cells, a multifunctional cytokine known to mediate the EMT (28), a process where cells lose epithelial markers and gain mesenchymal attributes allowing for a more mobile and migratory phenotype. TGF- $\beta$ is also pro-angiogenic, and along with vascular endothelial growth factor (VEGF), platelet-derived growth factor (PDGF), fibroblast growth factor, and SDF-1 is released by CAFs to enhance endothelial cell proliferation and migration (25).

The MDSC is a more recently described cell type that becomes activated in pathological states and has potent immunosuppressive 
capacity (29). In some pathologies, MDSCs help protect the host organ from the harmful effects of excessive immune stimulation. For example, MDSCs were elevated in mice with antigen-induced autoimmune enterocolitis, and their presence lead to a reduction of disease symptoms (30). In cancer states, however, activation of MDSCs is an effective means of protection from immunemediated killing (29) by inhibiting antigen-presenting dendritic cells, T and B cell proliferation, and natural killer cell cytotoxicity (31). Furthermore, MDSCs have also been implicated directly in tumor metastasis by helping to facilitate the EMT and to establish a distant premetastatic niche (PMN) (29).

\section{COMMUNICATION VIA EVs WITHIN THE TME}

The complex interactions displayed between cancer cells and the TME, as mentioned above, occur through a very complicated network of cellular communication. Many of these signaling pathways operate through direct cell-to-cell contact or using classical paracrine signaling loops of cytokines or growth factors with their receptors. However, more recently, EV shedding has emerged as another important mechanism of cellular cross-talk (7).

Extracellular vesicles are lipid bilayer-bound vehicles that are released from the cell membrane and carry nucleic acids (DNA, mRNA, and miRNA), proteins, and lipids to neighboring or distant cells (8). Although EVs were first described over 30 years ago as being released from reticulocytes (32), they have gained significant attention only recently as key factors in regulating both normal cell physiology and disease states. They now have been identified in nearly all eukaryotic cells (33) and prokaryotic cells (34) and have been isolated from most bodily fluids (8).

As shown in Figure 1, EVs are classified into two groups depending on their size, biogenesis, and method of release from the cell. Exosomes are $30-100 \mathrm{~nm}$ in diameter and are generated within large intracellular multivesicular bodies (35). They are released into the extracellular environment upon fusion with the plasma membrane.

Microvesicles (MVs) generally range from 100 to $1,000 \mathrm{~nm}$ and are formed when cell components travel to the plasma membrane to be released by membrane budding (36). Due to an incomplete understanding of exosome and MV biogenesis, and inconsistent methods of purification, the two terms are sometimes used interchangeably within the literature. The classical protocols for purification such as ultracentrifugation, density gradient centrifugation, and newer commercially available kits have been shown to co-isolate MVs and exosomes (37) as well as protein aggregates and other non-EV biomolecules that may interfere with EV specificity (38). Since current isolation methods are not yet standardized, it becomes difficult to assign specific functions to exosomes or MVs independently and why they are both included under the broad classification of EVs.

Extracellular vesicles exhibit wide-ranging roles in maintaining normal cellular and biological physiology. The EV lipid membrane protects its contents from enzyme degradation in

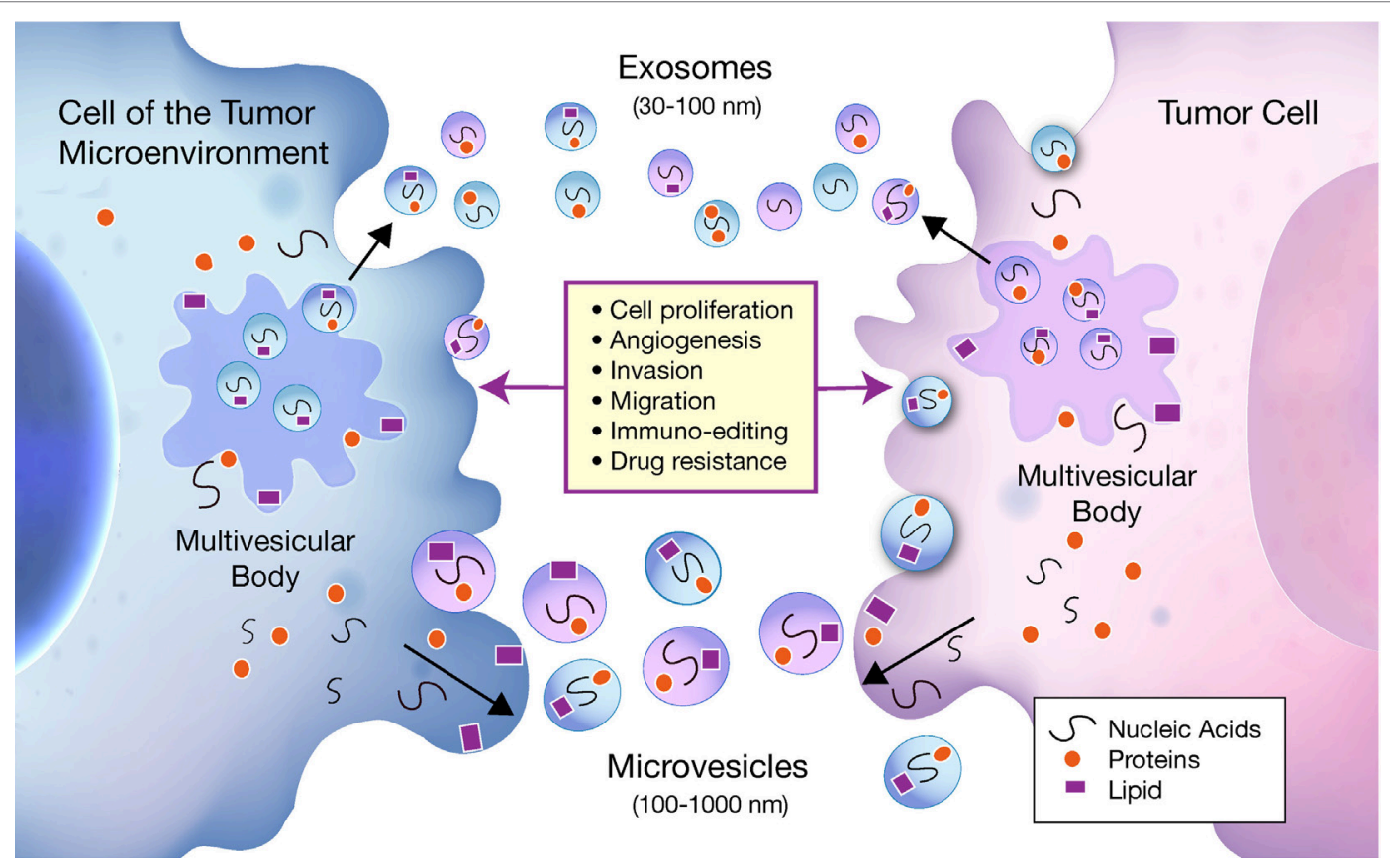

FIGURE 1 | Bidirectional communication occurs between tumor cells and cells of the tumor microenvironment (TME) via exosomes and microvesicles (MVs). Exosomes and MVs carry nucleic acids, proteins, and lipids between tumor cells and cells of the TME, which influence a multitude of pathways involved in tumor progression. Exosomes are 30-100 nm in diameter and are generated within larger intracellular multivesicular bodies. They are released into the extracellular environment on fusion with the plasma membrane. MVs generally range from 100 to $1000 \mathrm{~nm}$ and are formed when cell components travel to the plasma membrane to be released by membrane budding. 
bodily fluids making them ideal vessels to send material over a distance. They have been widely studied for their role in immune surveillance, blood coagulation, stem cell maintenance, tissue repair, and development (39). Since EVs play such a pivotal role in maintaining normal cell physiology, it is not surprising that their malfunctioning may lead to disease. EV involvement has been implicated in numerous pathological processes such as CCR5 receptor transport in HIV (40), beta amyloid transmission in Alzheimer's disease (41), and the spread of prion disease to neighboring cells (42). Undoubtedly, however, the most heavily researched area of EV-associated pathology is their role in tumor development and chemotherapeutic resistance in cancer.

\section{THE PLEOTROPIC ROLES OF EVs IN CANCER BIOLOGY}

Extracellular vesicles represent a common method of communication within the local TME and distant sites. Although research pertaining to EVs derived from tumor cells monopolizes the literature, more evidence is emerging of the significant roles of stromal cell-derived EVs in cancer biology. EVs isolated from both cell types have been implicated in various steps of tumor growth and development including cell proliferation, angiogenesis, migration, invasion, metastasis, immunoediting, and drug resistance. For a comprehensive list of the molecules involved in tumorigenesis carried by EVs from both cancer and non-cancer cells, which are mentioned in this article, see Table $\mathbf{1 .}$

\section{Proliferation}

Some of the first evidence of the involvement of EVs in proliferation was in glioblastoma (GBM) cell lines. U373 glioma cells that expressed a highly oncogenic form of epidermal growth factor receptor (EGFR), called EGFRvIII, had the ability to transmit this protein to non-EGFRvIII expressing cells via EVs. The recipient cell later expressed the mutated receptor leading to increased rates of proliferation (43). The study further confirmed that the cells expressing the malignant form of EGFR also had increased their rate of EV production to levels easily detected in the blood of its murine host. Later, additional studies also demonstrated the presence EGFRvIII mRNA within the vesicles (44), illustrating the diversity of EV cargo.

Tumor-derived EVs can also interact with and influence cells of the surrounding stroma. Breast cancer-derived EVs carry oncogenic proteins to surrounding fibroblasts inducing transformation and the acquisition of malignant features such as enhanced proliferation and survival (71). In other cancers, heat shock proteins (HSP70 and HSP90) and survivin, which are known to inhibit apoptosis and increase cellular proliferation, have been isolated from tumor-derived EVs and shown to promote a more aggressive cancer phenotype $(45,46)$.

Some of the important cells of the TME mediate their effect on tumor cells using EVs. For example, CAF-derived EVs containing increased levels of miRNA-21 suppress apoptosis and profoundly promote tumor growth in ovarian cancer (48). Also, increased pancreatic cancer aggressiveness was shown to be dependent on tumor cell-mediated uptake of CAF-derived Annexin A6+ (ANXA6) EVs, and while depletion of ANXA6 in CAFs impaired pancreatic tumor and metastasis occurrence, injection of CAFderived ANXA6+ EVs enhanced tumorigenesis (47). Human mesenchymal stem cells were also demonstrated to release EVs that lead to the phosphorylation of protein kinase B in gastric cancer cell lines and increased cancer cell proliferation (72).

\section{Angiogenesis}

As a tumor begins to proliferate, it requires increasing amounts of oxygen and nutrients to support the enlarging cellular population, making angiogenesis a necessary mechanism for tumor survival (73). EVs have been implicated in many of the sophisticated processes that allow angiogenesis to occur. In many cancer types, they have been specifically demonstrated to carry proangiogenic factors as cargo including VEGF, FGF2,

TABLE 1 | Molecules demonstrated to be transported by extracellular vesicles between cancer and non-cancerous cells.

\begin{tabular}{|c|c|c|}
\hline Category of molecule & Cancer cell derived & Non-cancer cell derived \\
\hline Proliferation & $\begin{array}{l}\text { EGFRvIlI }(43,44) \\
\text { HSP70, HSP90, Survivin }(45,46) \\
\text { Annexin A6+ }(47)\end{array}$ & miRNA-21 (48) \\
\hline Angiogenesis & $\begin{array}{l}\text { Vascular endothelial growth factor, FGF2, platelet-derived growth factor (49) } \\
\text { Centromere protein E, PDZ-binding kinase, cyclin-dependent kinase } 8 \text { (50) } \\
\text { miRNA-9 (49) }\end{array}$ & \\
\hline Epithelial-mesenchymal transition & Tumor necrosis factor- $\alpha$, interleukin 6 , Akt, $\beta$-catenin (51) & \\
\hline Migration/invasion/metastasis & $\begin{array}{l}\text { Transforming growth factor (TGF)- } \beta \text { (52) } \\
\text { MT1-metalloproteinase (53) } \\
\text { Fibronectin (54) } \\
\text { miR-181c (55) } \\
\text { Integrin alpha-V beta-5, integrin alpha- } 6 \text { beta-4, integrin alpha- } 6 \text { beta-1 (56) } \\
\text { Migration inhibitory factor (57) }\end{array}$ & $\begin{array}{l}\text { CD81 (58) } \\
\text { A disintegrin and metalloproteinase domain- } \\
\text { containing protein } 10(59)\end{array}$ \\
\hline Immunoediting & $\begin{array}{l}\text { TGF- } \beta(60,61) \\
\text { Fas ligand }(50,62-64) \\
\text { Galectin } 9(65)\end{array}$ & MHC class II molecules (66) \\
\hline
\end{tabular}


and PDGF (49). In fact, serum starvation and hypoxic conditions lead to a global increase in EV release from cancer cells (74).

Glioblastoma is characterized by severe hypoxia compared to many other cancer types (75), which results in large areas of necrosis. GBM cells have been demonstrated not only to secrete EVs containing tissue factor, a protein well known in the clotting cascade, but also shown to activate hypoxic endothelial cells leading to the formation of new blood vessels (76). Later studies of GBM also observed that hypoxic tumor-derived EVs significantly enhance angiogenesis both ex vivo and in vitro by stimulating endothelial cells to secrete soluble factors that trigger P13/AKT signaling in pericytes (77).

RNAs carried by EVs have also been implicated in neoangiogenic processes. In colorectal cancer, tumor-derived EVs containing cell cycle $\mathrm{M}$-phase-related mRNAs including centromere protein E, PDZ-binding kinase, and cyclin-dependent kinase 8 were demonstrated to promote endothelial proliferation (50). Furthermore, colorectal cancer cell-derived EVs have also been shown to carry miRNAs, including miRNA-9, which causes strong angiogenic effects by suppressing cytokine signaling 5 leading to enhanced activation of Janus kinase and thus driving endothelial cell migration (49).

\section{Cell Migration, Invasion, and Metastasis}

Cancer metastasis is a critical area of cancer biology as it leads to over $90 \%$ of solid tumor-related deaths (78). This complex mechanism involves multiple steps including invasion, intravasation into adjacent blood and lymph circulations, evasion of the host immune system, extravasation to distant organs, colonization, and finally the formation of micro- and macro-metastases. $\mathrm{EV}$ involvement in the metastatic process has been shown to be extensive as they orchestrate many of these pathological mechanisms.

As mentioned earlier, the CAF is a cell of the tumor stroma that is known to aid in the metastatic process through mechanisms inducing the activation of the EMT. EVs are utilized by tumor cells to help promote the transformation of fibroblasts to CAFs. Tumor-derived EVs from mesothelioma, prostate, bladder, and colorectal cancer cell lines containing TGF- $\beta$ triggered fibroblast differentiation into a CAF phenotype demonstrated by the expression of alpha-smooth muscle actin (52). Once transformed, CAFs may then also use EVs as a tool to encourage tumor cell migration and metastasis (59). Inducers of the EMT such as TGF- $\beta$, tumor necrosis factor alpha (TNF- $\alpha$ ), interleukin 6 , Akt, and $\beta$-catenin are also known cargo of tumor-derived EVs (51). Also, in orthotopic mouse models of breast cancer, EVs containing CD81 were released from CAFs and endocytosed by tumor cells, reloaded with the Wnt11 signaling factor, and re-released to the microenvironment. They then traveled to neighboring cancer cells causing cell polarization and directional motility (58). Another study reported that dermal fibroblasts expressing a CAF phenotype released EVs enriched in A disintegrin and metalloproteinase domain-containing protein 10, which can enhance breast cancer cell motility by activating RhoA and Notch signaling (59).

Extracellular matrix (ECM) remodeling is another important step for tumor invasion and subsequent metastasis to occur. EVs were noted to assist with ECM degradation through transport of metalloproteinases (MMPs) into the extracellular space giving room for cell migration to occur (53). It has also been demonstrated that fibrosarcoma- and melanoma-derived EVs containing MT1-MMP actively degraded type 1 collagen and gelatin (53). After extracellular space is created, tumor-derived EVs carrying ECM molecules such as fibronectin promote cell adhesion and assembly to influence cell directional motility (54).

Tominaga et al. found that the microRNA miR-181c was significantly upregulated in brain metastatic breast cancer cell-derived EVs. When tested in a blood-brain barrier model, miR-181c significantly downregulated the value of the transendothelial electrical resistance (55). They showed that EVs from brain metastatic cancer cells induce the abnormal localization of the tight junction proteins by transferring miR-181c into endothelial cells, which results in the destruction of cell-cell contact. This demonstrated that breast cancer-derived EVs can trigger the breakdown of the blood-brain barrier leading to brain metastasis.

In the 1800 s, Paget noticed that different tumor types tend to metastasize to specific organs leading to the "seed and soil" hypothesis of cancer metastasis (79). It is now well established that primary tumors can release cytokines, chemokines, and their receptors to direct metastatic cells to a preferred secondary site called the PMN (10). More recently discovered is that communication between the primary tumor and the PMN can be mediated through EVs. For example, a repertoire of integrins have been reported to guide the vesicles to specific organs. EVs expressing integrin alpha- $\mathrm{V}$ beta-5 specifically bind to Kupffer cells mediating liver metastasis, whereas integrin alpha- 6 beta- 4 and integrin alpha- 6 beta- 1 bind lung-resident fibroblasts and epithelial cells to mediate lung metastasis (56). Once EVs arrive at the predetermined distant site, their cargo is unloaded to aid in a stepwise creation of the PMN. For example, pancreatic cancer cell EVs were shown to travel to Kupffer cells in the liver to deliver macrophage migration inhibitory factor. This induced the secretion of TGF- $\beta$ in Kupffer cells and ultimately induced the recruitment of bone marrow-derived cells to complete PMN formation (57). Another important aspect of PMN formation is vascular leakiness, which facilitates the extravasation of malignant cells (80) via the delivery of specific molecules that trigger vessel permeabilization of endothelial cells (81) including those carried in EVs. In one study, human breast cancer-derived EVs promoted vascular leakiness in the lung by upregulating S100 proteins and activating Src kinase signaling (56).

\section{Immunoediting}

For tumor cells to progress and survive, they must evade the immune system. In the 1990s, it became established that EVs were used in normal immune cell physiological processes after studies illustrated that B-cells released EVs carrying MHC classII, co-stimulatory, and adhesion molecules that participate in the immune response (66). Later, studies began to elucidate the complex and dual roles of tumor-derived EVs as both immune activating and suppressing agents and therefore possessing antitumor and protumor properties (82). 
Tumor-derived EVs contain specific tumor antigens that may be enriched compared to the donor tumor cell. These antigens can be used as a source to create an enhanced immune response and lead to antitumor activities (83). In fact, this principle has been utilized in therapeutic strategies where dendritic cells from patients with malignant gliomas were exposed to tumor-derived EVs causing the induction of $\mathrm{CD}^{+}{ }^{+} \mathrm{T}$-cell-dependent antitumor effects (84). This idea of tumor- or immune cell-derived EVs being used as a cancer vaccine has led to multiple clinical trials (85-88).

Tumor-derived EVs, however, are also involved in multiple methods of immune suppression (89). Numerous studies have demonstrated that immunosuppressive regulatory T-cells (Tregs) can be generated, expanded, and activated by tumor-derived EVs (89). Mesothelioma-derived EVs with TGF- $\beta$ on the vesicle surface inhibited the proliferative response of $\mathrm{CD} 8^{+} \mathrm{T}$-cells to interleukin- 2 by increasing the number of Tregs (90). Likewise, EVs from colorectal cancer delivered TGF- $\beta$ to T-cells, which activated the Smad signaling pathway and changed their phenotype to that more similar to Treg cells (91). TGF- $\beta$ can also directly downregulate cells of the immune system. EVs isolated from acute myeloid leukemia and breast cancer cells were shown to suppress NK cells and T-cells, respectively, by delivering TGF- $\beta$ directly to the immune cell $(60,61)$.

Tumor-released miRNAs have more recently been studied as EV cargo causing immune suppression. While surrounded by the EV lipid bilayer, miRNAs are protected from degradation by RNase in the extracellular environment (92). One group studied contents of EVs released from nasopharyngeal carcinoma cells and reported five overexpressed miRNAs that, through inhibition of the MAP-kinase pathway, caused decreased proliferation in T-cells (93). Another group analyzed miRNA levels in dendritic cells that were treated with EVs from pancreatic cancer cells and found increased levels of 9 miRNAs that caused the downregulation of 200 mRNAs and a subsequent decrease in dendritic cell MHC class II expression (94). Furthermore, EV miRNA-cargo also suppresses immune cells by inducing $\mathrm{CD}^{+}$ T-cells to express a more Treg-like phenotype. This activity has been reported in multiple human cancer and mouse tumor models through the reduction of phosphatase and tensin homolog expression (95).

Fas ligand is a well-known protein of the TNF family that induces cell apoptosis on binding to its receptor. Multiple tumor types utilize Fas ligand to induce T-cell death as a means of immune escape (89). Some specific cancers such as melanoma, prostate, oral cancer, and colorectal cancer have demonstrated this ability by specifically releasing EVs containing Fas ligand directly to T-cells (50, 62-64). Galectin-9, like Fas ligand, also mediates apoptosis when bound to its receptor. In nasopharyngeal carcinoma, galactin- 9 was delivered to its receptor, Tim-3, on $\mathrm{CD} 4^{+}$Th1 helper cells in EVs causing cell death (65).

\section{Therapy Resistance}

The development of chemoradiation and targeted therapy resistance in cancer has remained a challenging hurdle to overcome for cancer treatment. Mechanisms including induction of salvage pathways, drug metabolism alterations, induction of the EMT, and enhanced DNA repair have all been implicated in resistance (96). Significant evidence is emerging that EVs may help facilitate a drug-resistant phenotype through two major methods. First, EVs can help deliver drug efflux pumps (DEPs) or other resistanceacquiring products from drug-resistance tumor cells to drugsensitive cells (97). Second, within the EV donor cells, DEPs can integrate into the EV itself and help sequester chemotherapeutic drugs to be released into the extracellular environment and leave the donor cell with sublethal concentrations (98).

Drug efflux pumps have long been acknowledged as a major contributor to multidrug resistance (MDR) in cancer, but their associations with EVs have only been recently explored. P-glycoprotein (P-gp) is one of the most well-studied DEPs. The first indication that P-gp was in the cargo of tumor-derived EVs was in leukemia cells. Drug-sensitive tumor cells were co-cultured with EVs isolated from drug-resistant cells, and after $4 \mathrm{~h}$ of incubation, the sensitive cells acquired a drug-resistant phenotype and were expressing functional P-gp (67). Other DEPs including MRP1, ABCG2, and ABCA3 are also transferred from resistant to susceptible cells by EVs leading to a MDR phenotype (68-70). Drug-resistant tumor cells can also transfer various other materials to susceptible cells using EVs. A multitude of RNAs including miRNAs, long non-coding RNAs, and functional mRNAs carried by EVs induced a MDR phenotype in previously drug-sensitive cells (99-101). Furthermore, irradiation of a variety of cancer cell lines was noted to cause increased absorption of EVs containing the protein survivin. These cancer cells experienced increased protection from toxic stressors and displayed increased proliferation and metastatic potential (102).

Extracellular vesicles can also be utilized by EV donor cells to aid in drug resistance. Previous studies have illustrated that decreases in $\mathrm{pH}$ may cause an increase in $\mathrm{EV}$ release from tumor cells (103). Some groups have proposed that within the acidic TME, alkaline drugs may be drawn into the acidic organelles of cancer cells and then eliminated from the cell by EV release (104106). In fact, it has been shown that EVs released from melanoma cells had cisplatin concentrations that negatively correlated with $\mathrm{pH}$. Furthermore, by using a human xenograft model, they found that treatment with a proton pump inhibitor, which raised the $\mathrm{pH}$ of the TME, lead to a decreased level of tumor-derived EV release and EVs containing decreased concentrations of cisplatin (107).

\section{THE DIAGNOSTIC VALUE OF EVs}

The early detection of cancer is perhaps one of the greatest reasons for a favorable survival (108). Therefore, it is important to investigate novel tools for earlier cancer identification. Since the contents of EVs reflect the cell from which they originate, detection of specific EVs in bodily fluids may have diagnostic potential (109). EVs have been successfully isolated from blood, urine, ascites, cerebro-spinal fluid, amniotic fluid, semen, saliva, and bile (8), and many display a cancer-specific signature that allows for easy detection. For example, EVs isolated from the ascites of colorectal cancer patients contain enriched levels of caludin-3 protein compared to non-pathologic samples (110). Patients with esophageal SCC have serum-isolated EVs enriched with miRNA-21 (111). The specific EV contents also represent a 
snapshot of the tumor cell status at the time of release. Therefore, they may help in the development of personalized therapeutics or even monitor the tumor's response to chemotherapy.

Ovarian cancer is known to present with vague and nonspecific symptoms. This unfortunate circumstance accounts for the majority of women becoming diagnosed with advanced disease and is why the average 5-year survival is less than $50 \%$ (3). Therefore, screening techniques that identify specific cancer biomarkers may be a very useful tool for early detection and a subsequent drop in mortality. One group demonstrated that higher concentrations of an array of miRNAs including miR-21 and miR-141 within EVs were also present in ovarian tumor tissue. Furthermore, higher levels of these miRNAs were associated with more advanced disease (112). Since then, studies have identified other ovarian cancer biomarkers including epithelial cell adhesion molecule and CD24 (113). Efforts are now being made to find practical and cost-effective methods for detecting cancer-specific EVs in patient blood or other bodily fluids.

Scalpel-free biopsies, also known as liquid biopsies, are novel methods for cancer detection. These techniques detect freefloating cancer cells, circulating tumor DNA, or EVs within bodily fluids. There have been varying antibody-based methodologies tested for analyzing EV proteins from bodily fluids without an EV isolation step, including flow cytometry, EV protein microarray, diagnostic magnetic resonance, and nanoplasmonic sensing technology (114). Although still far from standard practice, some companies have already produced commercial kits for detecting lung and prostate cancer where specific EVs are isolated and analyzed using chromatin immunoprecipitation assays (115). In colorectal cancer, an assay called "ExoScreen" can detect cancer-specific circulating double-positive (CD146/ CD9) EVs using photosensitive-beads from only $5 \mu$ l of patient serum (116).

\section{EV-CENTERED THERAPY}

Given the growing evidence of EV involvement in cancer pathogenesis, it seems intuitive to explore translational approaches that lead to their inhibition. Current studies are utilizing different techniques to inhibit vesicle formation, release, and cell uptake as well as blocking specific components of the EV. The drug amiloride has been shown in vivo to block secretion of tumor-derived EVs that contain membrane-associated heat shock protein 72 (HSP72) (117). HSP72 is constitutively expressed in many cancers and is associated with a poor prognosis (118). Furthermore, amiloride was shown to inhibit ceramide, an important mediator of EV biogenesis. Another drug, diannexin, inhibits phosphatidylserine, a regulator of cell adhesion, and EV endocytosis (119). Rab27 is a protein demonstrated to have a significant role in EV secretion (120). In highly metastatic mouse models of both melanoma and breast cancer, knocking down Rab27 led to a significant reduction of tumor EV production, primary tumor size, and metastasis (121, 122). However, since EVs are also essential participants in normal cell physiology, better techniques are required to distinguish and target pathological versus physiological EVs.

As mentioned previously, EVs have been used as cancer vaccines by carrying and providing tumor-specific antigens to immune cells, which prime the immune system and create a powerful immunological response against the tumor. Some of the first phase I clinical trials applying this methodology took place in melanoma and non-small-cell lung carcinoma where dendritic cell-derived EVs were loaded with MHC/tumor antigen and delivered back to patients $(86,87)$. These studies demonstrated that cancer vaccines are both feasible in creation and safe for administration. Later, EVs from the malignant ascites of colorectal cancer patients were isolated, mixed with specific cytokines, and administered back to the patient as a subcutaneous immunization. The investigators reported that combining ascites EVs with granulocyte-macrophage colony-stimulating factor induced specific antitumor cytotoxic T-lymphocyte activation (85). Furthermore, in a phase II clinical trial including patients with advanced small-cell-lung carcinoma, the administration of dendritic cell-derived EVs caused an increase in natural killer cell activity and longer progression-free survival for patients with low initial expression of natural cytotoxicity receptor NKp30 (88).

The realization that EVs are efficient vehicles for cell-to-cell communication has subsequently given rise to investigations of their use as a method for drug delivery. EVs display many potential advantages over current approaches. They are stable in serum, have specific cell-targeting capabilities, can overcome natural barriers such as the immune system or the bloodbrain barrier, and can deliver molecules such as miRNAs or siRNAs that are readily degraded in the serum (38). Multiple methods have been reported to successfully load EVs with a desired drug. Hydrophobic drugs have been demonstrated to integrate with EVs successfully by simply mixing and allowing the drug to pass through the EV lipid bilayer membrane (123). The loading of hydrophilic drugs has proven to be more challenging, but still possible by methods including electroporation, sonication, saponin-mediated permeabilization, and freeze-thaw cycles (124). Perhaps the most challenging aspect of EV-mediated drug deliver is the efficient targeting of specific cell types. Some groups have used transfection-based approaches to encourage cells to express organ-specific ligands or receptors that are loaded into EVs, released from the cell, and then isolated and collected for successive drug loading (124-126). Other groups are experimenting with iron oxide nanoparticles in combination with a drug within EVs to target specific areas of the body by the application of a magnetic field gradient (127).

\section{CONCLUSION}

Currently, there is a rich source of data that creates a powerful case for the involvement of EVs in most, if not all, aspects of tumor development and progression. The intimate association of EVs with cancer has exciting implications for both cancer diagnostics and therapeutics. However, our general knowledge of EVs and EV-mediated processes remains in its infancy compared to other fields of cancer biology. Perhaps the most important hurdle that is restraining progress is the inability to consistently isolate EV subtypes, preventing investigators from comprehensively comparing and assigning to them 
specific functions. EV subtype uncertainty is demonstrated in much of the literature when investigators use the broad term "extracellular vesicle" as opposed to "microvesicle" or "exosome." Isolation techniques cannot just focus on a single characteristic such as size or protein marker to discriminate among EV subtypes. Methods must use a combination of distinguishing features to better enrich the subpopulation, help define specific functions, and later to better categorize their role for therapeutics. Also, since EVs are key players in normal cell physiology, steps need to be taken to avoid interfering with normal processes. Despite current issues with EV isolation and subtyping, their role in cancer progression is dramatic and promises to become crucial in future advances in cancer diagnostics and therapeutics.

\section{REFERENCES}

1. Ferlay J, Soerjomataram I, Dikshit R, Eser S, Mathers C, Rebelo M, et al. Cancer incidence and mortality worldwide: sources, methods and major patterns in GLOBOCAN 2012. Int J Cancer (2015) 136(5):E359-86. doi:10.1002/ijc.29210

2. Siegel RL, Miller KD, Jemal A. Cancer statistics, 2016. CA Cancer J Clin (2016) 66(1):7-30. doi:10.3322/caac.21332

3. Howlader N, Noone AM, Krapcho M, Miller D, Bishop K, Altekruse SF, et al., editors. SEER Cancer Statistics Review, 1975-2013. Bethesda, MD: National Cancer Institute (2016). Available from: http://seer.cancer.gov/ csr/1975_2013/

4. Early Detection of Cancer. WHO (2017). Available from: http://www.who. int/cancer/detection/en/

5. Chen F, Zhuang X, Lin L, Yu P, Wang Y, Shi Y, et al. New horizons in tumor microenvironment biology: challenges and opportunities. BMC Med (2015) 13:45. doi:10.1186/s12916-015-0278-7

6. Quail DF, Joyce JA. Microenvironmental regulation of tumor progression and metastasis. Nat Med (2013) 19(11):1423-37. doi:10.1038/nm.3394

7. Raposo G, Stoorvogel W. Extracellular vesicles: exosomes, microvesicles, and friends. J Cell Biol (2013) 200(4):373-83. doi:10.1083/jcb.201211138

8. Colombo M, Raposo G, Thery C. Biogenesis, secretion, and intercellular interactions of exosomes and other extracellular vesicles. Annu Rev Cell Dev Biol (2014) 30:255-89. doi:10.1146/annurev-cellbio-101512-122326

9. Balkwill FR, Capasso M, Hagemann T. The tumor microenvironment at a glance. J Cell Sci (2012) 125(Pt 23):5591-6. doi:10.1242/jcs.116392

10. Joyce JA, Pollard JW. Microenvironmental regulation of metastasis. Nat Rev Cancer (2009) 9(4):239-52. doi:10.1038/nrc2618

11. Clark WH Jr, Elder DE, Guerry DT, Braitman LE, Trock BJ, Schultz D, et al. Model predicting survival in stage I melanoma based on tumor progression. J Natl Cancer Inst (1989) 81(24):1893-904. doi:10.1093/jnci/81.24.1893

12. Pages F, Berger A, Camus M, Sanchez-Cabo F, Costes A, Molidor R, et al. Effector memory T cells, early metastasis, and survival in colorectal cancer. N Engl J Med (2005) 353(25):2654-66. doi:10.1056/NEJMoa051424

13. Dunn GP, Bruce AT, Ikeda H, Old LJ, Schreiber RD. Cancer immunoediting: from immunosurveillance to tumor escape. Nat Immunol (2002) 3(11):991-8. doi:10.1038/ni1102-991

14. Grivennikov SI, Greten FR, Karin M. Immunity, inflammation, and cancer. Cell (2010) 140(6):883-99. doi:10.1016/j.cell.2010.01.025

15. Junttila MR, de Sauvage FJ. Influence of tumour micro-environment heterogeneity on therapeutic response. Nature (2013) 501(7467):346-54. doi:10.1038/nature 12626

16. Qian BZ, Pollard JW. Macrophage diversity enhances tumor progression and metastasis. Cell (2010) 141(1):39-51. doi:10.1016/j.cell.2010.03.014

17. Williams CB, Yeh ES, Soloff AC. Tumor-associated macrophages: unwitting accomplices in breast cancer malignancy. NPJ Breast Cancer (2016) 2:15025. doi:10.1038/npjbcancer.2015.25

18. Liu Y, Cao X. The origin and function of tumor-associated macrophages. Cell Mol Immunol (2015) 12(1):1-4. doi:10.1038/cmi.2014.83

\section{AUTHOR CONTRIBUTIONS}

RS, GM, JH, and LL made substantial contributions to conception and design; RS performed literature search, analyzed data, and wrote the manuscript; GM, XZ, CS, JH, DM, and LL contributed to critically revise the manuscript; JH and LL supervised the study and final approval of the version to be submitted.

\section{ACKNOWLEDGMENTS}

The authors would like to acknowledge the contribution of Ms. Barbara Siede for the medical illustration. This work is partially supported by the University of Queensland-Ochsner Seed Fund for Collaborative Research.

19. Biswas SK, Mantovani A. Macrophage plasticity and interaction with lymphocyte subsets: cancer as a paradigm. Nat Immunol (2010) 11(10):889-96. doi:10.1038/ni.1937

20. Franklin RA, Liao W, Sarkar A, Kim MV, Bivona MR, Liu K, et al. The cellular and molecular origin of tumor-associated macrophages. Science (2014) 344(6186):921-5. doi:10.1126/science. 1252510

21. Kalluri R, Zeisberg M. Fibroblasts in cancer. Nat Rev Cancer (2006) 6(5):392-401. doi:10.1038/nrc1877

22. De Wever O, Demetter P, Mareel M, Bracke M. Stromal myofibroblasts are drivers of invasive cancer growth. Int J Cancer (2008) 123(10):2229-38. doi:10.1002/ijc.23925

23. Kalluri R. The biology and function of fibroblasts in cancer. Nat Rev Cancer (2016) 16(9):582-98. doi:10.1038/nrc.2016.73

24. Li H, Fan X, Houghton J. Tumor microenvironment: the role of the tumor stroma in cancer. J Cell Biochem (2007) 101(4):805-15. doi:10.1002/jcb.21159

25. Shiga K, Hara M, Nagasaki T, Sato T, Takahashi H, Takeyama H. Cancerassociated fibroblasts: their characteristics and their roles in tumor growth. Cancers (Basel) (2015) 7(4):2443-58. doi:10.3390/cancers7040902

26. Orimo A, Gupta PB, Sgroi DC, Arenzana-Seisdedos F, Delaunay T, Naeem R, et al. Stromal fibroblasts present in invasive human breast carcinomas promote tumor growth and angiogenesis through elevated SDF-1/ CXCL12 secretion. Cell (2005) 121(3):335-48. doi:10.1016/j.cell.2005.02.034

27. Moatassim-Billah S, Duluc C, Samain R, Jean C, Perraud A, Decaup E, et al. Anti-metastatic potential of somatostatin analog SOM230: indirect pharmacological targeting of pancreatic cancer-associated fibroblasts. Oncotarget (2016) 7(27):41584-98. doi:10.18632/oncotarget.9296

28. Chaffer CL, Thompson EW, Williams ED. Mesenchymal to epithelial transition in development and disease. Cells Tissues Organs (2007) 185(1-3):7-19. doi:10.1159/000101298

29. Marvel D, Gabrilovich DI. Myeloid-derived suppressor cells in the tumor microenvironment: expect the unexpected. J Clin Invest (2015) 125(9) 3356-64. doi:10.1172/JCI80005

30. Haile LA, von Wasielewski R, Gamrekelashvili J, Kruger C, Bachmann O, Westendorf AM, et al. Myeloid-derived suppressor cells in inflammatory bowel disease: a new immunoregulatory pathway. Gastroenterology (2008) 135(3):871-81, 881.e1-5. doi:10.1053/j.gastro.2008.06.032

31. Almand B, Clark JI, Nikitina E, van Beynen J, English NR, Knight SC, et al. Increased production of immature myeloid cells in cancer patients: a mechanism of immunosuppression in cancer. J Immunol (2001) 166(1):678-89. doi:10.4049/jimmunol.166.1.678

32. Pan BT, Johnstone RM. Fate of the transferrin receptor during maturation of sheep reticulocytes in vitro: selective externalization of the receptor. Cell (1983) 33(3):967-78. doi:10.1016/0092-8674(83)90040-5

33. Lee Y, El Andaloussi S, Wood MJ. Exosomes and microvesicles: extracellular vesicles for genetic information transfer and gene therapy. Hum Mol Genet (2012) 21(R1):R125-34. doi:10.1093/hmg/dds317

34. Ellis TN, Kuehn MJ. Virulence and immunomodulatory roles of bacterial outer membrane vesicles. Microbiol Mol Biol Rev (2010) 74(1):81-94 doi:10.1128/MMBR.00031-09 
35. Dragovic RA, Gardiner C, Brooks AS, Tannetta DS, Ferguson DJ, Hole P, et al. Sizing and phenotyping of cellular vesicles using nanoparticle tracking analysis. Nanomedicine (2011) 7(6):780-8. doi:10.1016/j.nano.2011.04.003

36. Tkach $\mathrm{M}$, Thery $\mathrm{C}$. Communication by extracellular vesicles: where we are and where we need to go. Cell (2016) 164(6):1226-32. doi:10.1016/j. cell.2016.01.043

37. Thery C, Amigorena S, Raposo G, Clayton A. Isolation and characterization of exosomes from cell culture supernatants and biological fluids. Curr Protoc Cell Biol (2006) 3:3.22. doi:10.1002/0471143030.cb0322s30

38. Verma M, Lam TK, Hebert E, Divi RL. Extracellular vesicles: potential applications in cancer diagnosis, prognosis, and epidemiology. BMC Clin Pathol (2015) 15:6. doi:10.1186/s12907-015-0005-5

39. Andaloussi SEL, Mager I, Breakefield XO, Wood MJ. Extracellular vesicles: biology and emerging therapeutic opportunities. Nat Rev Drug Discov (2013) 12(5):347-57. doi:10.1038/nrd3978

40. Mack M, Kleinschmidt A, Bruhl H, Klier C, Nelson PJ, Cihak J, et al. Transfer of the chemokine receptor CCR5 between cells by membrane-derived microparticles: a mechanism for cellular human immunodeficiency virus 1 infection. Nat Med (2000) 6(7):769-75. doi:10.1038/77498

41. Bellingham SA, Guo BB, Coleman BM, Hill AF. Exosomes: vehicles for the transfer of toxic proteins associated with neurodegenerative diseases? Front Physiol (2012) 3:124. doi:10.3389/fphys.2012.00124

42. Emmanouilidou E, Melachroinou K, Roumeliotis T, Garbis SD, Ntzouni M, Margaritis LH, et al. Cell-produced alpha-synuclein is secreted in a calcium-dependent manner by exosomes and impacts neuronal survival. J Neurosci (2010) 30(20):6838-51. doi:10.1523/ JNEUROSCI.5699-09.2010

43. Al-Nedawi K, Meehan B, Micallef J, Lhotak V, May L, Guha A, et al. Intercellular transfer of the oncogenic receptor EGFRvIII by microvesicles derived from tumour cells. Nat Cell Biol (2008) 10(5):619-24. doi:10.1038/ncb1725

44. Skog J, Wurdinger T, van Rijn S, Meijer DH, Gainche L, Sena-Esteves M, et al. Glioblastoma microvesicles transport RNA and proteins that promote tumour growth and provide diagnostic biomarkers. Nat Cell Biol (2008) 10(12):1470-6. doi:10.1038/ncb1800

45. Graner MW, Cumming RI, Bigner DD. The heat shock response and chaperones/heat shock proteins in brain tumors: surface expression, release, and possible immune consequences. JNeurosci (2007) 27(42):11214-27. doi:10.1523/JNEUROSCI.3588-07.2007

46. Khan S, Jutzy JM, Aspe JR, McGregor DW, Neidigh JW, Wall NR. Survivin is released from cancer cells via exosomes. Apoptosis (2011) 16(1):1-12. doi:10.1007/s10495-010-0534-4

47. Leca J, Martinez S, Lac S, Nigri J, Secq V, Rubis M, et al. Cancer-associated fibroblast-derived annexin A6+ extracellular vesicles support pancreatic cancer aggressiveness. J Clin Invest (2016) 126(11):4140-56. doi:10.1172/ JCI87734

48. Au Yeung CL, Co NN, Tsuruga T, Yeung TL, Kwan SY, Leung CS, et al. Exosomal transfer of stroma-derived miR21 confers paclitaxel resistance in ovarian cancer cells through targeting APAF1. Nat Commun (2016) 7:11150. doi:10.1038/ncomms 11150

49. Wendler F, Favicchio R, Simon T, Alifrangis C, Stebbing J, Giamas G. Extracellular vesicles swarm the cancer microenvironment: from tumorstroma communication to drug intervention. Oncogene (2017) 36(7):877-84. doi:10.1038/onc.2016.253

50. Huber V, Fais S, Iero M, Lugini L, Canese P, Squarcina P, et al. Human colorectal cancer cells induce T-cell death through release of proapoptotic microvesicles: role in immune escape. Gastroenterology (2005) 128(7): 1796-804. doi:10.1053/j.gastro.2005.03.045

51. Vella LJ. The emerging role of exosomes in epithelial-mesenchymaltransition in cancer. Front Oncol (2014) 4:361. doi:10.3389/fonc.2014.00361

52. Webber J, Steadman R, Mason MD, Tabi Z, Clayton A. Cancer exosomes trigger fibroblast to myofibroblast differentiation. Cancer Res (2010) 70(23):9621-30. doi:10.1158/0008-5472.CAN-10-1722

53. Hakulinen J, Sankkila L, Sugiyama N, Lehti K, Keski-Oja J. Secretion of active membrane type 1 matrix metalloproteinase (MMP-14) into extracellular space in microvesicular exosomes. J Cell Biochem (2008) 105(5):1211-8. doi:10.1002/jcb.21923

54. Sung BH, Ketova T, Hoshino D, Zijlstra A, Weaver AM. Directional cell movement through tissues is controlled by exosome secretion. Nat Commun (2015) 6:7164. doi:10.1038/ncomms8164
55. Tominaga N, Kosaka N, Ono M, Katsuda T, Yoshioka Y, Tamura K, et al. Brain metastatic cancer cells release microRNA-181c-containing extracellular vesicles capable of destructing blood-brain barrier. Nat Commun (2015) 6:6716. doi: $10.1038 /$ ncomms7716

56. Hoshino A, Costa-Silva B, Shen TL, Rodrigues G, Hashimoto A, Tesic Mark M, et al. Tumour exosome integrins determine organotropic metastasis. Nature (2015) 527(7578):329-35. doi:10.1038/nature15756

57. Costa-Silva B, Aiello NM, Ocean AJ, Singh S, Zhang H, Thakur BK, et al. Pancreatic cancer exosomes initiate pre-metastatic niche formation in the liver. Nat Cell Biol (2015) 17(6):816-26. doi:10.1038/ncb3169

58. Luga V, Zhang L, Viloria-Petit AM, Ogunjimi AA, Inanlou MR, Chiu E, et al. Exosomes mediate stromal mobilization of autocrine Wnt-PCP signaling in breast cancer cell migration. Cell (2012) 151(7):1542-56. doi:10.1016/j. cell.2012.11.024

59. Shimoda M, Principe S, Jackson HW, Luga V, Fang H, Molyneux SD, et al. Loss of the Timp gene family is sufficient for the acquisition of the CAF-like cell state. Nat Cell Biol (2014) 16(9):889-901. doi:10.1038/ncb3021

60. Szczepanski MJ, Szajnik M, Welsh A, Whiteside TL, Boyiadzis M. Blast-derived microvesicles in sera from patients with acute myeloid leukemia suppress natural killer cell function via membrane-associated transforming growth factor-beta1. Haematologica (2011) 96(9):1302-9. doi:10.3324/haematol.2010.039743

61. Rong L, Li R, Li S, Luo R. Immunosuppression of breast cancer cells mediated by transforming growth factor-beta in exosomes from cancer cells. Oncol Lett (2016) 11(1):500-4. doi:10.3892/ol.2015.3841

62. Andreola G, Rivoltini L, Castelli C, Huber V, Perego P, Deho P, et al. Induction of lymphocyte apoptosis by tumor cell secretion of FasL-bearing microvesicles. J Exp Med (2002) 195(10):1303-16. doi:10.1084/jem.20011624

63. Abusamra AJ, Zhong Z, Zheng X, Li M, Ichim TE, Chin JL, et al. Tumor exosomes expressing Fas ligand mediate CD8+ T-cell apoptosis. Blood Cells Mol Dis (2005) 35(2):169-73. doi:10.1016/j.bcmd.2005.07.001

64. Kim JW, Wieckowski E, Taylor DD, Reichert TE, Watkins S, Whiteside TL. Fas ligand-positive membranous vesicles isolated from sera of patients with oral cancer induce apoptosis of activated T lymphocytes. Clin Cancer Res (2005) 11(3):1010-20.

65. Klibi J, Niki T, Riedel A, Pioche-Durieu C, Souquere S, Rubinstein E, et al. Blood diffusion and Th1-suppressive effects of galectin-9-containing exosomes released by Epstein-Barr virus-infected nasopharyngeal carcinoma cells. Blood (2009) 113(9):1957-66. doi:10.1182/blood-2008-02-142596

66. Raposo G, Nijman HW, Stoorvogel W, Liejendekker R, Harding CV, Melief CJ, et al. B lymphocytes secrete antigen-presenting vesicles. J Exp Med (1996) 183(3):1161-72. doi:10.1084/jem.183.3.1161

67. Bebawy M, Combes V, Lee E, Jaiswal R, Gong J, Bonhoure A, et al. Membrane microparticles mediate transfer of P-glycoprotein to drug sensitive cancer cells. Leukemia (2009) 23(9):1643-9. doi:10.1038/leu.2009.76

68. Lu JF, Luk F, Gong J, Jaiswal R, Grau GE, Bebawy M. Microparticles mediate MRP1 intercellular transfer and the re-templating of intrinsic resistance pathways. Pharmacol Res (2013) 76:77-83. doi:10.1016/j.phrs.2013.07.009

69. Ifergan I, Scheffer GL, Assaraf YG. Novel extracellular vesicles mediate an ABCG2-dependent anticancer drug sequestration and resistance. Cancer Res (2005) 65(23):10952-8. doi:10.1158/0008-5472.CAN-05-2021

70. Aung T, Chapuy B, Vogel D, Wenzel D, Oppermann M, Lahmann M, et al. Exosomal evasion of humoral immunotherapy in aggressive B-cell lymphoma modulated by ATP-binding cassette transporter A3. Proc Natl Acad Sci U S A (2011) 108(37):15336-41. doi:10.1073/pnas.1102855108

71. Antonyak MA, Li B, Boroughs LK, Johnson JL, Druso JE, Bryant KL, et al. Cancer cell-derived microvesicles induce transformation by transferring tissue transglutaminase and fibronectin to recipient cells. Proc Natl Acad Sci U S A (2011) 108(12):4852-7. doi:10.1073/pnas.1017667108

72. Gu H, Ji R, Zhang X, Wang M, Zhu W, Qian H, et al. Exosomes derived from human mesenchymal stem cells promote gastric cancer cell growth and migration via the activation of the Akt pathway. Mol Med Rep (2016) 14(4):3452-8. doi:10.3892/mmr.2016.5625

73. Carmeliet P, Jain RK. Angiogenesis in cancer and other diseases. Nature (2000) 407(6801):249-57. doi:10.1038/35025220

74. King HW, Michael MZ, Gleadle JM. Hypoxic enhancement of exosome release by breast cancer cells. BMC Cancer (2012) 12:421. doi:10.1186/ 1471-2407-12-421

75. Evans SM, Judy KD, Dunphy I, Jenkins WT, Hwang WT, Nelson PT, et al. Hypoxia is important in the biology and aggression of human glial brain 
tumors. Clin Cancer Res (2004) 10(24):8177-84. doi:10.1158/1078-0432. CCR-04-1081

76. Svensson KJ, Kucharzewska P, Christianson HC, Skold S, Lofstedt T, Johansson MC, et al. Hypoxia triggers a proangiogenic pathway involving cancer cell microvesicles and PAR-2-mediated heparin-binding EGF signaling in endothelial cells. Proc Natl Acad Sci U S A (2011) 108(32):13147-52. doi:10.1073/pnas.1104261108

77. Kucharzewska P, Christianson HC, Welch JE, Svensson KJ, Fredlund E, Ringner $\mathrm{M}$, et al. Exosomes reflect the hypoxic status of glioma cells and mediate hypoxia-dependent activation of vascular cells during tumor development. Proc Natl Acad Sci U S A (2013) 110(18):7312-7. doi:10.1073/ pnas. 1220998110

78. Valastyan S, Weinberg RA. Tumor metastasis: molecular insights and evolving paradigms. Cell (2011) 147(2):275-92. doi:10.1016/j.cell.2011.09.024

79. Paget S. The distribution of secondary growths in cancer of the breast. Cancer Metastasis Rev (1989) 8(2):98-101.

80. Huang Y, Song N, Ding Y, Yuan S, Li X, Cai H, et al. Pulmonary vascular destabilization in the premetastatic phase facilitates lung metastasis. Cancer Res (2009) 69(19):7529-37. doi:10.1158/0008-5472.CAN-08-4382

81. Becker A, Thakur BK, Weiss JM, Kim HS, Peinado H, Lyden D. Extracellular vesicles in cancer: cell-to-cell mediators of metastasis. Cancer Cell (2016) 30(6):836-48. doi:10.1016/j.ccell.2016.10.009

82. Zeelenberg IS, Ostrowski M, Krumeich S, Bobrie A, Jancic C, Boissonnas A, et al. Targeting tumor antigens to secreted membrane vesicles in vivo induces efficient antitumor immune responses. Cancer Res (2008) 68(4):1228-35. doi:10.1158/0008-5472.CAN-07-3163

83. Robbins PD, Morelli AE. Regulation of immune responses by extracellular vesicles. Nat Rev Immunol (2014) 14(3):195-208. doi:10.1038/nri3622

84. Bu N, Wu H, Sun B, Zhang G, Zhan S, Zhang R, et al. Exosome-loaded dendritic cells elicit tumor-specific CD8+ cytotoxic $\mathrm{T}$ cells in patients with glioma. J Neurooncol (2011) 104(3):659-67. doi:10.1007/s11060-011-0537-1

85. Dai S, Wei D, Wu Z, Zhou X, Wei X, Huang H, et al. Phase I clinical trial of autologous ascites-derived exosomes combined with GM-CSF for colorectal cancer. Mol Ther (2008) 16(4):782-90. doi:10.1038/mt.2008.1

86. Escudier B, Dorval T, Chaput N, Andre F, Caby MP, Novault S, et al. Vaccination of metastatic melanoma patients with autologous dendritic cell (DC) derived-exosomes: results of the first phase I clinical trial. J Transl Med (2005) 3(1):10. doi:10.1186/1479-5876-3-10

87. Morse MA, Garst J, Osada T, Khan S, Hobeika A, Clay TM, et al. A phase I study of dexosome immunotherapy in patients with advanced non-small cell lung cancer. J Transl Med (2005) 3(1):9. doi:10.1186/1479-5876-3-9

88. Besse B, Charrier M, Lapierre V, Dansin E, Lantz O, Planchard D, et al. Dendritic cell-derived exosomes as maintenance immunotherapy after first line chemotherapy in NSCLC. Oncoimmunology (2016) 5(4):e1071008. doi:10.1080/2162402x.2015.1071008

89. Czernek L, Duchler M. Functions of cancer-derived extracellular vesicles in immunosuppression. Arch Immunol Ther Exp (Warsz) (2017) 65(4):311-23. doi:10.1007/s00005-016-0453-3

90. Clayton A, Mitchell JP, Court J, Mason MD, Tabi Z. Human tumor-derived exosomes selectively impair lymphocyte responses to interleukin-2. Cancer Res (2007) 67(15):7458-66. doi:10.1158/0008-5472.CAN-06-3456

91. Yamada N, Kuranaga Y, Kumazaki M, Shinohara H, Taniguchi K, Akao Y. Colorectal cancer cell-derived extracellular vesicles induce phenotypic alteration of T cells into tumor-growth supporting cells with transforming growth factor-beta1-mediated suppression. Oncotarget (2016) 7(19):27033-43. doi:10.18632/oncotarget.7041

92. Koga Y, Yasunaga M, Moriya Y, Akasu T, Fujita S, Yamamoto S, et al. Exosome can prevent RNase from degrading microRNA in feces.J Gastrointest Oncol (2011) 2(4):215-22. doi:10.3978/j.issn.2078-6891.2011.015

93. Ye SB, Li ZL, Luo DH, Huang BJ, Chen YS, Zhang XS, et al. Tumor-derived exosomes promote tumor progression and $\mathrm{T}$-cell dysfunction through the regulation of enriched exosomal microRNAs in human nasopharyngeal carcinoma. Oncotarget (2014) 5(14):5439-52. doi:10.18632/oncotarget.2118

94. Diao J, Yang X, Song X, Chen S, He Y, Wang Q, et al. Exosomal Hsp70 mediates immunosuppressive activity of the myeloid-derived suppressor cells via phosphorylation of Stat3. Med Oncol (2015) 32(2):453. doi:10.1007/ s12032-014-0453-2

95. Yin Y, Cai X, Chen X, Liang H, Zhang Y, Li J, et al. Tumor-secreted miR-214 induces regulatory $\mathrm{T}$ cells: a major link between immune evasion and tumor growth. Cell Res (2014) 24(10):1164-80. doi:10.1038/cr.2014.121
96. Azmi AS, Bao B, Sarkar FH. Exosomes in cancer development, metastasis, and drug resistance: a comprehensive review. Cancer Metastasis Rev (2013) 32(3-4):623-42. doi:10.1007/s10555-013-9441-9

97. Jaiswal R, Raymond Grau GE, Bebawy M. Cellular communication via microparticles: role in transfer of multidrug resistance in cancer. Future Oncol (2014) 10(4):655-69. doi:10.2217/fon.13.230

98. Gong J, Luk F, Jaiswal R, George AM, Grau GE, Bebawy M. Microparticle drug sequestration provides a parallel pathway in the acquisition of cancer drug resistance. Eur J Pharmacol (2013) 721(1-3):116-25. doi:10.1016/j. ejphar.2013.09.044

99. Xiao X, Yu S, Li S, Wu J, Ma R, Cao H, et al. Exosomes: decreased sensitivity of lung cancer A549 cells to cisplatin. PLoS One (2014) 9(2):e89534. doi:10.1371/journal.pone.0089534

100. Takahashi K, Yan IK, Kogure T, Haga H, Patel T. Extracellular vesiclemediated transfer of long non-coding RNA ROR modulates chemosensitivity in human hepatocellular cancer. FEBS Open Bio (2014) 4:458-67. doi:10.1016/j.fob.2014.04.007

101. Lai CP, Kim EY, Badr CE, Weissleder R, Mempel TR, Tannous BA, et al. Visualization and tracking of tumour extracellular vesicle delivery and RNA translation using multiplexed reporters. Nat Commun (2015) 6:7029. doi:10.1038/ncomms8029

102. Khan S, Aspe JR, Asumen MG, Almaguel F, Odumosu O, AcevedoMartinez S, et al. Extracellular, cell-permeable survivin inhibits apoptosis while promoting proliferative and metastatic potential. Br J Cancer (2009) 100(7):1073-86. doi:10.1038/sj.bjc.6604978

103. Parolini I, Federici C, Raggi C, Lugini L, Palleschi S, De Milito A, et al. Microenvironmental $\mathrm{pH}$ is a key factor for exosome traffic in tumor cells. J Biol Chem (2009) 284(49):34211-22. doi:10.1074/jbc.M109.041152

104. Raghunand N, Martinez-Zaguilan R, Wright SH, Gillies RJ.pH and drug resistance. II. Turnover of acidic vesicles and resistance to weakly basic chemotherapeutic drugs. Biochem Pharmacol (1999) 57(9):1047-58. doi:10.1016/S0006-2952(99)00021-0

105. Raghunand N, He X, van Sluis R, Mahoney B, Baggett B, Taylor CW, et al. Enhancement of chemotherapy by manipulation of tumour $\mathrm{pH}$. Br J Cancer (1999) 80(7):1005-11. doi:10.1038/sj.bjc.6690455

106. Ouar Z, Lacave R, Bens M, Vandewalle A. Mechanisms of altered sequestration and efflux of chemotherapeutic drugs by multidrug-resistant cells. Cell Biol Toxicol (1999) 15(2):91-100. doi:10.1023/A:1007521430236

107. Federici C, Petrucci F, Caimi S, Cesolini A, Logozzi M, Borghi M, et al. Exosome release and low $\mathrm{pH}$ belong to a framework of resistance of human melanoma cells to cisplatin. PLoS One (2014) 9(2):e88193. doi:10.1371/ journal.pone. 0088193

108. American Cancer Society. Cancer Prevention \& Early Detection Facts \& Figures 2015-2016. Atlanta: American Cancer Society (2015).

109. Yanez-Mo M, Siljander PR, Andreu Z, Zavec AB, Borras FE, Buzas EI, et al. Biological properties of extracellular vesicles and their physiological functions. J Extracell Vesicles (2015) 4:27066. doi:10.3402/jev.v4.27066

110. Choi DS, Park JO, Jang SC, Yoon YJ, Jung JW, Choi DY, et al. Proteomic analysis of microvesicles derived from human colorectal cancer ascites. Proteomics (2011) 11(13):2745-51. doi:10.1002/pmic.201100022

111. Tanaka Y, Kamohara H, Kinoshita $\mathrm{K}$, Kurashige J, Ishimoto $\mathrm{T}$, Iwatsuki M, et al. Clinical impact of serum exosomal microRNA-21 as a clinical biomarker in human esophageal squamous cell carcinoma. Cancer (2013) 119(6):1159-67. doi:10.1002/cncr.27895

112. Taylor DD, Gercel-Taylor C. MicroRNA signatures of tumor-derived exosomes as diagnostic biomarkers of ovarian cancer. Gynecol Oncol (2008) 110(1):13-21. doi:10.1016/j.ygyno.2008.04.033

113. Liang B, Peng P, Chen S, Li L, Zhang M, Cao D, et al. Characterization and proteomic analysis of ovarian cancer-derived exosomes. J Proteomics (2013) 80:171-82. doi:10.1016/j.jprot.2012.12.029

114. Fujita Y, Yoshioka Y, Ochiya T. Extracellular vesicle transfer of cancer pathogenic components. Cancer Sci (2016) 107(4):385-90. doi:10.1111/cas.12896

115. Chi KR. The tumour trail left in blood. Nature (2016) 532(7598):269-71. doi:10.1038/532269a

116. Yoshioka Y, Kosaka N, Konishi Y, Ohta H, Okamoto H, Sonoda H, et al. Ultrasensitive liquid biopsy of circulating extracellular vesicles using ExoScreen. Nat Commun (2014) 5:3591. doi:10.1038/ncomms4591

117. Chalmin F, Ladoire S, Mignot G, Vincent J, Bruchard M, Remy-Martin JP, et al. Membrane-associated Hsp72 from tumor-derived exosomes mediates STAT3-dependent immunosuppressive function of mouse and human 
myeloid-derived suppressor cells. JClin Invest (2010) 120(2):457-71. doi:10.1172/JCI40483

118. Yaglom JA, Gabai VL, Sherman MY. High levels of heat shock protein Hsp72 in cancer cells suppress default senescence pathways. Cancer Res (2007) 67(5):2373-81. doi:10.1158/0008-5472.can-06-3796

119. Lima LG, Chammas R, Monteiro RQ, Moreira ME, Barcinski MA. Tumor-derived microvesicles modulate the establishment of metastatic melanoma in a phosphatidylserine-dependent manner. Cancer Lett (2009) 283(2):168-75. doi:10.1016/j.canlet.2009.03.041

120. Ostrowski M, Carmo NB, Krumeich S, Fanget I, Raposo G, Savina A, et al. Rab27a and Rab27b control different steps of the exosome secretion pathway. Nat Cell Biol (2010) 12(1):19-30; sup pp 1-13. doi:10.1038/ncb2000

121. Peinado H, Lavotshkin S, Lyden D. The secreted factors responsible for pre-metastatic niche formation: old sayings and new thoughts. Semin Cancer Biol (2011) 21(2):139-46. doi:10.1016/j.semcancer.2011.01.002

122. Bobrie A, Krumeich S, Reyal F, Recchi C, Moita LF, Seabra MC, et al. Rab27a supports exosome-dependent and -independent mechanisms that modify the tumor microenvironment and can promote tumor progression. Cancer Res (2012) 72(19):4920-30. doi:10.1158/0008-5472.can-12-0925

123. Vader P, Mol EA, Pasterkamp G, Schiffelers RM. Extracellular vesicles for drug delivery. Adv Drug Deliv Rev (2016) 106(Pt A):148-56. doi:10.1016/j. addr.2016.02.006

124. Haney MJ, Klyachko NL, Zhao Y, Gupta R, Plotnikova EG, He Z, et al. Exosomes as drug delivery vehicles for Parkinson's disease therapy. J Control Release (2015) 207:18-30. doi:10.1016/j.jconrel.2015.03.033

125. Wiklander OP, Nordin JZ, O'Loughlin A, Gustafsson Y, Corso G, Mager I, et al. Extracellular vesicle in vivo biodistribution is determined by cell source, route of administration and targeting. J Extracell Vesicles (2015) 4:26316. doi:10.3402/jev.v4.26316

126. Tian Y, Li S, Song J, Ji T, Zhu M, Anderson GJ, et al. A doxorubicin delivery platform using engineered natural membrane vesicle exosomes for targeted tumor therapy. Biomaterials (2014) 35(7):2383-90. doi:10.1016/j. biomaterials.2013.11.083

127. Silva AK, Luciani N, Gazeau F, Aubertin K, Bonneau S, Chauvierre C, et al. Combining magnetic nanoparticles with cell derived microvesicles for drug loading and targeting. Nanomedicine (2015) 11(3):645-55. doi:10.1016/j. nano.2014.11.009

Conflict of Interest Statement: The authors declare that the research was conducted in the absence of any commercial or financial relationships that could be construed as a potential conflict of interest.

The reviewer, EP, and handling editor declared their shared affiliation, and the handling editor states that the process nevertheless met the standards of a fair and objective review.

Copyright (๔) 2017 Sullivan, Maresh, Zhang, Salomon, Hooper, Margolin and Li. This is an open-access article distributed under the terms of the Creative Commons Attribution License (CC BY). The use, distribution or reproduction in other forums is permitted, provided the original author(s) or licensor are credited and that the original publication in this journal is cited, in accordance with accepted academic practice. No use, distribution or reproduction is permitted which does not comply with these terms. 